\title{
Opinion: A Prototype for a Computerized National Mammography Registry and Tracking System Using Telecommunications and the Internet
}

\begin{abstract}
In this report, we analyze the feasability and discuss the potential benefits of using currently available technology for the wide-area registration and tracking of mammography patients. In our prototype, three dissimilar computer systems transmitted mammographic data (demographics and the results of mammograms) in a standardized format to a central data repository. Two of the three systems were dedicated computerized mammography systems and one was a general-purpose radiology information system. Highspeed modems and the Internet were used to connect with the central repository, which could be queried in real time by remote users. Our results indicated that a busy mammography practice, using the slowest transmission method we tested (14-kilobaud modem), could transmit several days of mammographic data to a central repository in a matter of minutes. To implement systems that provide nation-wide mammographic tracking and follow up, more in-depth planning, development, and testing are necessary. Copyright 11995 by W.B. Saunders Company
\end{abstract}

Mark S. Frank
KEY WORDS: breast cancer, mammography, widearea networks, medical information systems, outcomes analysis, computer data base.

$\mathbf{P}$ ERSONAL COMPUTERS (PCs) are useful for many of the data-processing tasks encountered in a mammography practice. Advantages include faster dissemination of mammographic results, automated generation of correspondence, facilitation of tracking patients who have had (or should have) mammograms, drastic decreases in redundant data entry, decreased needs for ancillary personnel, and facilitation of clinical research, ${ }^{1-5}$ all serving to improve patient care and reduce the risk of malpractice. ${ }^{6-7}$ Some computerized mammography data-base systems have been integrated with or built into radiology information systems ${ }^{8}$ or hospital information systems. However, some additional benefits can be realized by developing a mammographic registry and tracking system on a much broader geographic scope. Using the Internet and inexpensive telecommunications equipment, we have developed and tested methods for transferring mammographic data between dissimilar computer systems and storing the information on an Internet-based computer, thus establishing an infrastructure to make this data available (via modem or Internet) for several potentially useful purposes including the nation-wide tracking of patients, epidemiologic research, and quality assurance.

\section{MATERIALS AND METHODS}

Our prototype was composed of three different computer systems that could connect to a central data repository by using either a modem, the Internet, or both (Fig 1). One of the systems was a Macintosh (Apple Computer Corp, Cupertino, CA) executing a mammography tracking and follow-up system that was programmed in FoxPro (Microsoft Corp, Redmond, WA). This system serves as an adjunct to our general-purpose radiology information system, IDXrad (IDX Inc, Burlington, VT). Locally developed software transfers data pertaining to mammograms and breast sonograms from IDXrad to the FoxPro system whenever the mammographer desires to synchronize the data on the two systems. The second system was an IBM PC-compatible computer running a stand-alone mammographic reporting and tracking system that was programmed in FoxBase (Microsoft). The largest implementation of this system has been in use since 1987 with a census of approximately 60,000 patients. ${ }^{9}$ The third system was an independently operated IDXrad system in use at a medical center affiliated with ours. Using the Internet, locally developed software transmitted mammographic results in real time via a virtual socket-to-socket connection from this remote IDXrad system to our VAX 4600 minicomputer (Digital Equipment Corp, Maynard, MA).

Our VAX served as the central data repository for the prototype. All data were transferred to the repository in health-level 7 (HL7) format. ${ }^{10}$ The preexisting software on the Macintosh and IBM-compatible systems was upgraded so that each system could convert data from FoxPro and FoxBase formats, respectively, into HL7 format on a periodic basis (eg, daily or weekly), thus enabling the en masse transfer of data from newly processed examinations to the repository. Conversion into HL7 format was a feature already available on the remote IDXrad system. Data were transferred from the Macintosh system to the repository using an Internet connection with file transfer protocol (FTP). On the IBM-compatible system, a 14.4-kilobaud modem was used to transfer mammographic data using the ZModem protocol during an interactive session on the

From the Department of Radiology, the University of Washington School of Medicine, Seattle, WA.

Address reprint requests to Mark S. Frank, MD, Department of Radiology, University of Washington School of Medicine, Harborview Medical Center, 325 9th Ave, Seattle, WA 98104.

Copyright 11995 by W.B. Saunders Company

0897-1889/95/0803-0003\$3.00/0 


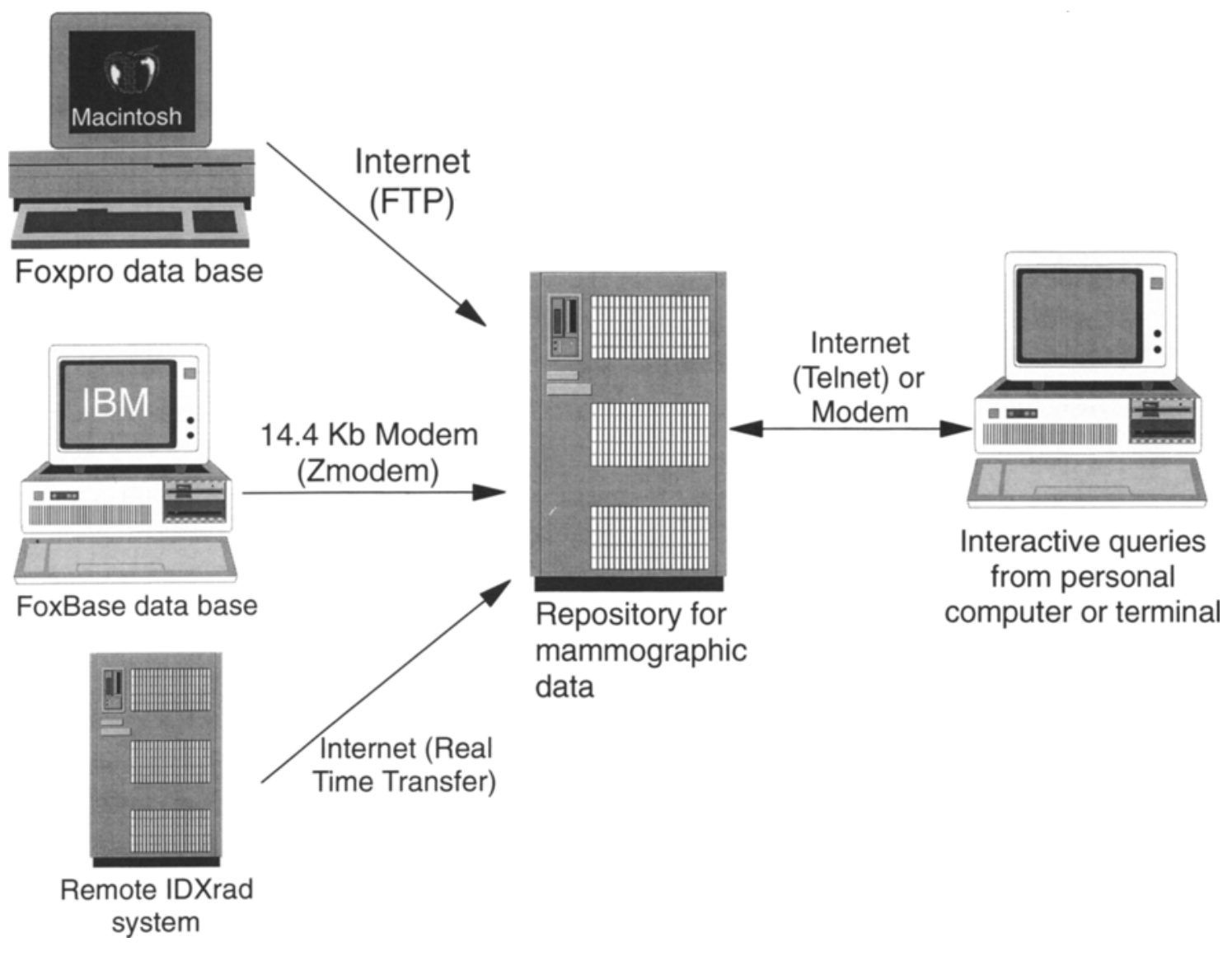

Fig 1. Schematic overview of the prototype system described by the authors is shown.

VAX. The HL7 messages were compressed with no loss of data before transfer via modem to maximize throughput. A continuously running program on the VAX automatically recognized the arrival of new data from either of these systems, decompressed the data if necessary, and incorporated it into the repository. An important distinction is that any transmission of data to the repository from the Macintosh or IBM-compatible systems required periodic interaction by the users of those systems, whereas transfer from the remote IDXrad system occurred automatically, and information arriving from that system was added to the repository's data base as it arrived in real time.

The repository on our VAX was a data base created and maintained with the Record Management Services component of the VAX/VMS operating system (Digital Equipment Corp). The software used to maintain the repository supported simultaneous read and write access to the data base for multiple users. The data were indexed by identification number and name for each patient. Information stored about the patient and the mammographic examination are listed in Table 1 . A password-protected account was created to provide interactive access to the repository. By dialing into the VAX with a modem or connecting over the Internet with a Telnet session, the user could query the repository using a patient's name or identification number. The system would list examinations on file for the patient and display information about the patient, the results of the mammograms, and the practice where the mammograms were obtained. Users were not allowed to modify the data.

\section{RESULTS}

The length of transmitted HL7 messages was typically between 1 and $4 \mathrm{kB}$. Transmission speed over the Internet connection from the

Table 1. Information Transmitted to the Mammographic Data Repository

\footnotetext{
Identification number of patient

Name, sex, and data of birth of patient

Address and home and work telephone numbers of patient Social security number (if available)

Mammographer's name

Address and telephone number of mammographer (if available)

Type of examination

Date of examination

Relevant clinical history

Technologist (if available)

Report of mammographic interpretation

Encoded outcome of mammographic examination (if available)
} 
remote IDXrad system was approximately 30 $\mathrm{kB}$ per second (eg, 7.5 to $30 \mathrm{HL} 7$ messages per second). Transmission speed when using FTP on the Macintosh, which required less network routing to access our VAX, was approximately $80 \mathrm{kB} / \mathrm{s}$. The effective transmission speed using the modem on the IBM-compatible system was approximately $4 \mathrm{kB} / \mathrm{s}$ (although modem speed was approximately $1.5 \mathrm{kB} / \mathrm{s}$, the typical compression ratio attained with HL7 data was slightly less than 3:1). As anticipated, this was by far the slowest transmission speed encountered for any method tested, yet still provided a throughput of 1 to 4 HL7 messages per second. Our results suggest that a busy mammography practice that performed 150 mammograms per day, using a modem, would on a worst-case basis transmit a day's data in approximately 150 seconds (2.5 minutes). The response time for queries on the repository was virtually instantaneous, even when the data base was loaded with several thousand records of simulated data (approximately 100 Mbyte).

\section{DISCUSSION}

A computerized national mammography registry and tracking system offers several potential advantages. For example, whenever a patient moves to a new location or changes physicians, the information regarding the patient's mammographic history would be immediately available when she reports, as a new patient, for her next mammogram. This would often be helpful because patients frequently cannot remember where their prior mammograms were done or who interpreted them. The radiologist could use his or her PC to immediately retrieve prior mammographic data for that patient. This would instantly update the radiologist's data base, provide the results of prior mammograms, and show the name, location, and phone number of the patient's prior radiologists so that the prior mammograms can be obtained for comparison as soon as possible. The immediate availability of this information would expedite the interpretation cycle for the radiologist, thus shortening the period of time that a patient must wait to receive final results of her mammogram. Someday, if digital mammography and high-speed, widearea networks are implemented on a nation-wide basis, even the prior mammographic images would be immediately available for comparison.
Large-scale public-health studies would benefit tremendously from access to a good-quality database that contained consistent information about millions of patients. In fact, such a database is essential for answering questions that are focused on populations, and might help resolve some of the controversies regarding early detection and treatment of breast cancer. Furthermore, a mammography registry is an ideal cornerstone for a national breast cancer registry. For example, the data for patients with positive biopsy samples could be easily transferred to a national breast cancer registry because biopsy results would be included in the data transferred from the mammographer's computer system to the national mammographic tracking system.

Our intent was to explore the technical feasibility of developing a national mammography registry and tracking system using currently available technology. Although our results indicate that there are no major technologic obstacles, some interesting problems were immediately apparent in our prototype. For example, two of the systems contributing to the repository identified patients by using a hospital identification number, whereas patients from the other system were identified with their social security number. If one patient had been processed on any two of the three mammographic systems that contributed to the repository, that patient would have appeared (by identification number) to have been two different people. Thus, to serve a regional or nation-wide population, there is clearly a need to establish a standardized system for uniquely identifying patients. ${ }^{11}$ Currently, the most promising method is to use social security numbers, but this apparently straightforward option is not without controversy.

Another issue that must be addressed is confidentiality of patients' medical data. The Internet is intentionally a minimally secured network. Network "snoopers" with the appropriate equipment can spy on transmitted data, unbeknownst to the sender or recipient. Recently, the Internet has gained much attention as a potential conduit for commerce. ${ }^{12-14} \mathrm{Be}$ cause personal financial information must be protected from unauthorized access, methods are being developed to encrypt and securely transfer confidential data on the Internet. The same, or similar, encryption technology can be 
used with confidential medical information. Similarly, interactive access to a repository would need to be restricted so that only authorized users (eg, mammographers needing to know the results or whereabouts of prior examinations) could execute a query. If personal identifiers (eg, name, identification number, addresses) were excluded, data could be transferred to ancillary data bases for epidemiologic research without a breech in confidentiality.

In our experiment, two of the mammography data-base systems transmitted codified information to the repository indicating the outcome of each mammogram (eg, mammographer's overall impression regarding likelihood of malignancy, recommended follow up, and interval of time until follow-up occurs). The other system (IDXrad) transmitted the entire mammographic report, but did not transmit encoded results of the mammogram. The most effective implementation of a nation-wide tracking system would likely use a standardized method for encoding much of the information regarding mammographic examinations, including the outcomes of mammograms and biopsies, before transmitting data to a repository rather than transmitting free-form textual data. This would substantially decrease transmission times and storage requirements, and is essential for consistent retrospective analyses. Establishing a national standard for codification would require cooperation among commercial vendors of computerized mammography data-base systems, authors of noncommercial systems, and vendors of general-purpose radiology information systems. The lexicon of the American College of Radiology's Breast Imaging, Reporting, and Data System would be an excellent model from which to derive many of the necessary codes. ${ }^{15}$

In addition to a need for standards in codification, there are also needs to determine which data would be transmitted to the repository and to specify in some detail the structure of transmitted HL7 data. Fortunately, we found that a relatively small effort was necessary to make an existing computerized mammography data-base system capable of generating and exporting
HL7-formatted data, suggesting that the cost of achieving compliance should be minimal for vendors of commercial mammographic software. It seems logical to initially develop a repository that would receive and store only a germane subset of the data maintained on commercial mammographic systems, yet design the repository with capabilities to expand and adapt to new needs as they occur. Thus, in its initial implementation, a national mammographic tracking system would store (1) information to identify and locate a patient and the patient's prior mammograms and mammographers, (2) encoded historic information regarding risk factors and prior breast surgery, and (3) encoded data regarding the outcomes of prior mammograms (eg, mammographer's impression and recommendation), breast sonograms, and biopsies.

There are many potential benefits to be realized from a national mammography registry and tracking system. Although such a system seems technically feasible, perhaps the greatest challenges are the administrative and political issues regarding such a huge data base containing confidential medical information. The initiative to create and maintain such a system should arise from organizations with nation-wide authority such as the American College of Radiology, the National Cancer Institute, or both. There will be costs for developing and maintaining what in all likelihood would be a distributed data base containing many gigabytes of information about millions of patients. Policy would need to be established regarding how the data is made available for clinical and research purposes. Methods would need to be developed to assure the integrity and security of the data. Nevertheless, as our national information infrastructure is upgraded and the cost of digital technology continues to decline, we should begin to address (and solve) the administrative and political problems that might encumber the development of a national mammography registry and tracking system so that patients will ultimately receive the highest quality of care possible.

\section{REFERENCES}

1. Sickles EA: The usefulness of computers in managing the operation of a mammography screening practice. AJR 155:755-761, 1990
2. Sickles EA: The use of computers in mammography screening. Radiol Clin N Am 25:1015-1030, 1987

3. Haug PJ, Tocino IM, Clayton PD, et al: Automated 
management of screening and diagnostic mammography. Radiology 164:747-752, 1987

4. Dunne MG, Dunne SB: Automated mammographic reporting and follow-up with an inexpensive lap-top microcomputer. J Digit Imaging 2:147-149, 1989

5. Frank MS: Computerized mammography data base systems. AJR 161:287-290, 1993

6. Monticciolo DL, Sickles EA: Computerized follow-up of abnormalities detected at mammography screening. AJR 155:751-753, 1990

7. Harris RP, O’Malley MS, Fletcher SW, et al: Prompting physicians for preventive procedures: A five-year study of manual and computer reminders. Am J Prev Med $6: 145-152,1990$

8. Frank MS, Johnson JA: Computerized tracking of mammography patients: Value of a radiology information system integrated with a personal computer data base. AJR 163:705-708, 1994
9. Rubin E, Frank MS, Stanley RJ, et al: Patient-initiated mobile mammography: Analysis of the patients and the problems. S Med J 83:178-184, 1990

10. Hettinger BJ, Brazile RP: Health Level Seven (HL7): Standard for healthcare electronic data transmissions. Comput Nurs 12:13-16, 1994

11. Bidgood WD, Tracy WR: In search of the name. Proceedings of the Annual Symposium of Computer Applications in Medical Care, 1993, pp 54-58

12. Knowles A: Security chip, interface aim to assist electronic commerce. PC-Week 12:15, 1995

13. Arnum E: The move to electronic commerce gets under way. Business Communications Review 24:28, 1994

14. Corcoran E: Microsoft, Visa team up on electronic commerce; new software would allow sales via Internet. Washington Post 117:B4, 1994

15. Kopans DB: Standardized mammography reporting. Radiol Clin N Am 30:257-264, 1992 